\title{
Belphégor
}

\section{Meesters, Gert, Pâques, Frédéric et Vrydaghs, David (s. la dir. de), Les Métamorphoses de Spirou}

\section{C[h]ris Reyns}

\section{(2) OpenEdition}

1 Journals

\section{Electronic version}

URL: https://journals.openedition.org/belphegor/2149

DOI: 10.4000/belphegor.2149

ISSN: 1499-7185

Publisher

LPCM

\section{Electronic reference}

C[h]ris Reyns, "Meesters, Gert, Pâques, Frédéric et Vrydaghs, David (s. la dir. de), Les Métamorphoses de Spirou", Belphégor [Online], 18-1 | 2020, Online since 15 January 2020, connection on 29 June 2021. URL: http://journals.openedition.org/belphegor/2149 ; DOI: https://doi.org/10.4000/belphegor.2149

This text was automatically generated on 29 June 2021.

\section{(c) (i) $(9)$}

Belphégor est mis à disposition selon les termes de la Licence Creative Commons Attribution - Pas d'Utilisation Commerciale - Pas de Modification 4.0 International. 


\section{Meesters, Gert, Pâques, Frédéric et Vrydaghs, David (s. la dir. de), Les Métamorphoses de Spirou}

\section{C[h]ris Reyns}

\section{REFERENCES}

Les Métamorphoses de Spirou, Gert Meesters, Frédéric Pâques et David Vrydaghs (s. la dir. de), Liège : Presses Universitaires de Liège, Collection ACME, 2019, 200p.

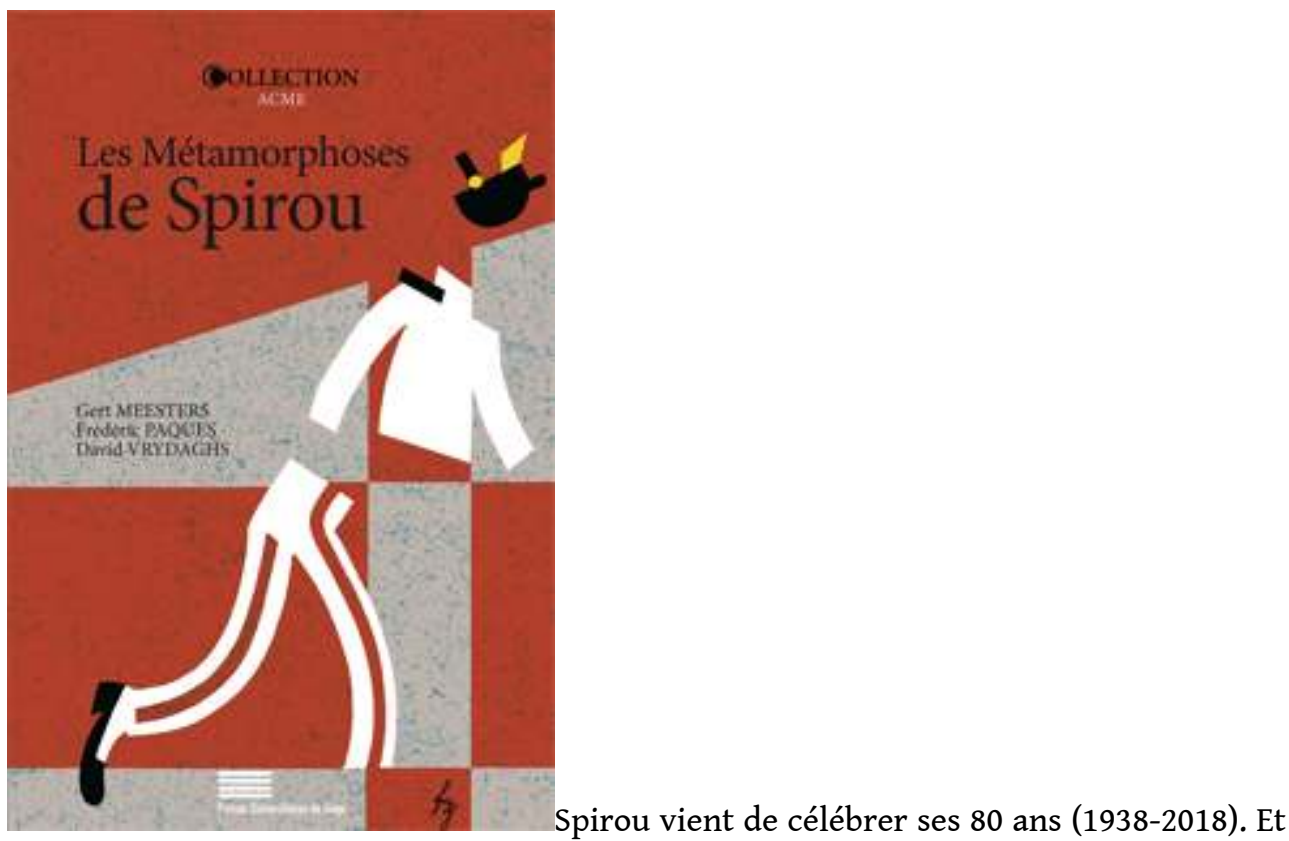
quel plus beau cadeau lui faire que de lui offrir une reconnaissance académique, qui 
plus est, de qualité, tant dans sa matérialité (papier glacé, nombreuses illustrations, superbes couleurs, reliure solide) que par le haut niveau critique de ses contributions.

1 Pour le lecteur non-francophone et qui ne connaîtrait pas ce personnage, il faut d'abord rappeler que Spirou, autant que Tintin, est un héros très populaire dans le domaine francophone; ensuite, qu'en plus de la série mère qui contient 55 albums, il y a quelques hors-séries et une série parallèle («Le Spirou de ...» de 14 albums à cette date); il y a aussi des séries non officielles reconnues par l'éditeur telles des pastiches par Chaland, des parodies, des réécritures de style « roman graphique » et des préquels ... Au total plus de 120 histoires complètes qui continuent à relativement bien se vendre aujourd'hui. Il faudrait encore ajouter : un parc Spirou (Monteux, Belgique, depuis juin 2018), un film (2018), et une collaboration avec l'ONU (Spirou4Rights, 2018)!

2 Or, cette série constitue un cas unique dans le domaine francophone pour ce qui est de la question de l'auctorialité. Premièrement, comme les trois « directeurs » de ce volume le notent dans leur brève introduction et les collaborateurs dans presque chaque chapitre, Spirou fut créé par plusieurs auteurs, puis repris par de nombreux auteursdessinateurs et/ou scénaristes dont Franquin est sans doute le plus connu. Deuxièmement, même lorsqu'un seul nom apparaissait sur la couverture, de nombreux collaborateurs contribuaient à la création, tels des «donneurs d'idées ", des décoristes, des coloristes, ... (103). Conséquemment, ces aventures «se singularisent par leur hétérogénéité graphique et scénaristique » (7). Troisièmement, elle est propriété des éditions Dupuis, ce qui est un cas rare dans la BD franco-belge (par contraste avec la tradition étatsunienne où cela est plutôt la norme). D'où le titre « les métamorphoses de Spirou » avec en plus "un clin d'œil au travail réalisé par [le chercheur et polygraphe] Jean-Marie Apostolidés à propos de Tintin [Les Métamorphoses de Tintin, 1984]» (8).

On constatera dès la lecture de l'introduction et de la table des matières de ce $2^{\mathrm{e}}$ volume du groupe ACME (le premier portait sur L'Association) que comme très souvent dans les études francophones de la BD, c'est l'analyse formelle qui domine, héritage de la sémiotique. Il comprend toutefois des contributions originales qui se distinguent de cette tradition. Fait de treize chapitres de longueur assez variable, le volume est divisé en deux parties. L'une porte sur des analyses macroscopiques de la dynamique d'ensemble de la série et l'autre (9 chapitres) porte davantage sur l'étude d'un auteur ou même d'un album (et donc plutôt des études microscopiques). Même si la notion d'héritage présentée par les trois directeurs ne semble pas évidente pour certains des chapitres, on voit bien comment, en dépit d'une grande diversité, ils ont cherché à mettre en scène cette notion traitée parfois implicitement, mais d'autres fois très explicitement (e.g., chapitre 12 par Crucifix et Moura).

Dick Tomasovic commence son chapitre par ce qui pourrait être une synthèse de tout l'ouvrage et même de toutes les aventures de Spirou. C'est un gag de sept cases de la série Le Petit Spirou (1990) contenant aventure, suspense, humour, émotion, des Spirou dans l'eau, sur terre, dans l'air, avec ses différents ennemis (Zorglub, animaux sauvages, Zantafio, tribus « sauvages »), l'obligeant à « bondir, courir, plonger, tomber, ...» et «le condamnant au mouvement perpétuel pour sa survie» (12). Cette mise en images du mouvement-vitesse dans un médium fixe n'est pas unique et est en fait typique du $19^{\mathrm{e}}$ et plus encore du $20^{\mathrm{e}}$ siècle (ce que Paul Virilio, ici cité, a bien analysé). Elle se retrouve dans de nombreuses BD (des débuts avec Töpffer aux cartoons animés et aux superhéros), mais elle a pourtant ses particularités. Ce mouvement est visible 
graphiquement, mais aussi à travers le motif du voyage. Tomasovic nous donne de belles analyses d'images, comme par exemple celle de cette fameuse "naissance de Spirou » par Rob-Vel dont la dernière case utilise les lignes de vitesse (17) et où le chien coupé en deux « vient suggérer le travail de mise en mouvement par l'ellipse propre à la $\mathrm{BD}$ » (17). Cette importance du mouvement est bien illustrée dans le choix de la "composition promotionnelle pour les 75 ans de Spirou en 2013 " où les huit représentations de Spirou par huit auteurs différents mettent aussi en évidence cette unité : ils sont tous en mouvement. Somme toute avec peu de lignes de mouvement et de vitesse, et juste le dessin du corps (les bras et jambes), prolongé par la technologie (voiture, avion, coptère, robot, ...) et les animaux (Spip, Marsupilami), on voit comment le personnage a été influencé par le dessin animé, le cinéma burlesque et le cinéma d'action. Comme le conclut Tomasovic, si le conservatisme de Spirou n'est pas « un agent du déséquilibre » (23) dans une société elle-même en déséquilibre représentée par le savant fou, il ne peut pourtant pas être simplement effacé par la fin, le plus souvent conservatrice. Car, comme dans le burlesque, il doit rester quelque chose de cette énergie qui s'oppose à l'Ordre. C'est d'ailleurs aussi ce que David Turgeon dit dans le chapitre suivant en "pointant du doigt les dictatures (se proposant même de les renverser [...], corrigeant par-là une vision colonialiste autrefois colportée par Franquin ", 26).

5 Le chapitre 2, de David Turgeon, est un des textes les plus courts et les moins académiques, avec ses commentaires souvent incisifs mais mêlés à des jugements parfois un peu hâtifs, et un style fait de nombreuses parenthèses et de points d'exclamation et d'interrogation. Cependant, il présente une perspective très intéressante et plutôt rare dans le monde francophone, non celle du critique savant ou de l'auteur mais celle du fan, ou plutôt de l'auteur comme fan, d'où son titre « Spirou et ses lecteurs ». Ainsi, « la chance de Spirou, c'est d'avoir vu son nom lié à un journal qui a su traverser la Seconde Guerre mondiale", où le jeune Franquin recrée un personnage qui n'avait " rien de commun avec celui de Rob-Vel que le nom et l'allure » (31), lui qui l'avait inventé comme "mascotte» (31).On citera encore la brève comparaison entre Panade à Champignac et Les Bijoux de la Castafiore (et même Alph'art) où Panade " impose une relecture pour tout dire parfaitement iconoclaste, de son propre système » (33). Enfin la reprise par de nombreux « lecteurs »-créateurs montre combien les albums de Franquin constituaient non seulement une œuvre riche d'inventions mais aussi une œuvre ouverte aux réinterprétations, dont certaines (Fournier, Bravo) sont dignes du maître Franquin.

6 «L'aventure en héritage » (chapitre 3, 35-53) de David Vrydaghs, est un des chapitres les plus longs. Après avoir réinscrit le récit d'aventure dans un contexte plus large, celui du $19^{\mathrm{e}}$ siècle, comme l'avait montré Matthieu Letourneux, et après avoir insisté sur l'inscription plus accentuée de l'humour dans Spirou, Vrydaghs divise son chapitre en quatre parties inégales portant sur quatre étapes-auteurs : Franquin, Fournier, Tome et Janry, et Yoann et Vehlmann. Vrydaghs montre les choix que vont faire les auteurs dans les possibilités offertes par la série, comme le rythme soutenu ou interrompu par des gags, le fait que Spirou sera tour à tour fort ou faible, seul ou à égalité avec Fantasio, central ou marginalisé (par le Marsupilami par exemple), ou encore les variations de style (plus léger ou plus grave). Dans la partie Franquin (37-43), à travers une comparaison avec Tintin (personnage, journal et aventure), il ressort que Spirou est un "gauchissement» du héros d'aventure (38). Cependant, même si Franquin semble préférer l'anti-héros (e.g., Gaston), ces héros gauchis comme Spirou « n'hésitent 
pas non plus à courir de grands risques pour secourir un ami ou une créature en danger » (40). C'est ici que l'importance de l'humour se montre cruciale. Influencé par les débuts de sa carrière comme animateur, puis par la concurrence avec Tintin (journal et personnage), Franquin glisse d'un humour burlesque à un comique de situation (40). La deuxième partie porte sur Fournier (44-46) qui hérite de Franquin, son maitre, mais se distingue aussi de lui. Avec Fournier, l'aventure retrouve un ton humoristique classique sans discontinuer d'album en album, et sans interruption dans chaque album au rythme soutenu. Il remplace le Marsupilami par le magicien japonais Itoh, plus intégré au rythme de l'histoire, et il utilise aussi des personnages plus héroïques. Dans la troisième partie portant sur les albums de Tome et Janry (46-52), comme le souligne le sous-titre, l'aventure est plus en noir (mort) et rouge (sang, violence), ce qui est aussi reflété par les teintes plus sombres ou plus criardes, et le plus grand réalisme des auteurs. Mais «le taux faible des ventes de La Frousse aux trousses (1988), la mort de Vandooren qui les soutenait et une certaine lassitude ont provoqué l'abandon de la série par ces auteurs » (49). Avec eux, Spirou était aussi plus proche du personnage plus fort et plus sûr de Fournier et plus central, comme sa «vacance » de Machine qui rêve le montre (52). La dernière partie, brève (moins d'une page), portant sur Yoann et Vehlmann, montre que ceux-ci rétablissent une connexion avec Franquin dès leur première aventure située à Champignac, avec le protagoniste à nouveau habillé en groom, et le fait qu'il ne se maîtrise plus trop (53).

Le chapitre 4 de Gert Meesters (55-67, + 3 pages de graphes) est une étude des techniques narratives ou des procédés stylistiques sur un plan macroscopique. Avec des critères relativement précis dans la sélection des éléments significatifs, comme le choix représentatif des auteurs et des histoires, l'équilibre entre images et textes ou le nombre de cases, Meesters montre qu'il peut définir les grandes tendances qui caractérisent les auteurs choisis. Les résultats confirment souvent ce que l'on savait déjà d'eux, sur certains points bien expliqués par les intertitres («Les multiples RobVel »; « une transition en douceur de Jijé à Franquin»; "Franquin, auteur en mouvement», "Fournier, l'importance du texte»). Cependant, ces résultats macroscopiques complexifient aussi les idées reçues sur d'autres points (e.g., l'évolution de Tome et Janry est inverse de celle de Franquin). Les deux dernières pages sont malheureusement moins convaincantes puisque ces analyses macroscopiques sont basées sur une seule histoire pour chaque dernière série (64-65). Cette technique a déjà été utilisée par Meesters avec des collaborateurs (Forgeville et El Refaie, 2014), mais aussi par Scott McCloud (1993, non-repris dans la bibliographie finale) et par Neil Cohn (2013, cité dans ce chapitre et dans la bibliographie). Si ces références sont utiles, je ne suis pas sûr de toutes les comprendre. Même si elles cherchent toutes à renforcer un point important, i.e., que dans tout langage (et la BD en est un), des évolutions s'effectuent en dehors du contrôle individuel, la référence à la "main invisible " d'Adam Smith me semble équivoque. D'une part, elle est un peu trop rapide, quand on sait combien cette notion est problématique, et d'autre part, elle est inutile, puisque la linguistique a démontré cela depuis plus de cent ans, bien avant le livre de Rudi Keller (1994) et sans besoin d'établir de rapport avec Adam Smith. En fait, les tables en fin de chapitre suffiraient, tant elles sont claires et utiles pour ce genre de lecture macroscopique. Comme le met en évidence la conclusion, cette lecture réintroduit une perspective plus distancée dans les études "littéraires ", déjà proposée en littérature par la « lecture à distance » de Franco Moretti et plus récemment par Bart Beaty pour les comics. 
8 Le bref chapitre 5 d'Olivier Odaert, intitulé « Préhistoire de Spirou » (71-80), est clair et instructif, même s'il est quelque peu redondant. Odaert nous rappelle d'abord la complexité de l'histoire de la naissance et des premiers développements de Spirou qui chronologiquement irait de l'influence de l'Américain Branner (auteur de Winnie Winkle [Bicot en français]) à la création de son Toto, puis de son Spirou. Il nous présente une analyse formelle qui met en évidence les modes de lecture, opposant deux sens de lectures complémentaires : l'une traditionnelle, de gauche à droite, et une autre différente, soit de droite à gauche, soit circulaire (Toto, 77), les deux rendant la lecture de cette BD tabulaire plutôt que linéaire. On peut toutefois regretter que le chapitre répète plusieurs fois ce qui a déjà été dit (et que l'auteur signale d'ailleurs à plusieurs reprises) par Tomasovic au premier chapitre, insistant sur le mouvement, ce qui fait perdre de vue l'autre élément intéressant du chapitre, le jeu avec le mouvement-trajet, qui sera aussi traité par Glaude au chapitre 7 (96).

9 Le chapitre 6, par Frédéric Pâques, s'intitule «Quand Jijé dessinait Spirou» (81-94). Après avoir reconnu que Franquin est l'auteur le plus important de Spirou, Pâques met ici en évidence le rôle clé de Jijé. Celui-ci a en effet été crucial avec, par exemple, l'introduction de Fantasio, personnage qui contribue à déstructurer le récit, comme le montre l'analyse d'une planche datant de 1945 (83). Ceci prouve une fois encore que l'évolution de la BD n'a nullement été linéaire, du gaufrier aux déstructurations diverses des Fred et autres comics et mangas d'aujourd'hui, comme on a parfois tendance à le croire. On le voit encore dans le fait que ses deux derniers récits (juillet 45 et mars 46) font un retour au gaufrier mais sont aussi influencés par le dessin animé en général et en particulier celui d'un Tex Avery. Chose remarquable, Pâques nous montre que Franquin a sans doute en fait influencé Jijé dans ses dernières histoires (86). Il fait aussi la lecture d'une BD moins connue et des plus intéressantes, «Comme une mouche au plafond ", qui reprend certains éléments déjà présentés par Tomasovic, mais en y ajoutant de nouveaux, comme celui de ne pas donner d'explication «scientifique » au mystère de Spirou marchant sur le plafond.

Le chapitre 7, de Benoît Glaude ( Franquin dialoguiste de Spirou et Fantasio, entre la tradition de Jijé et l'innovation de Greg ", 95-112), est le seul à s'attaquer aux dialogues, alors que les autres chapitres s'intéressent à la narration ou à l'image. Glaude rappelle la complexité de l'attribution des rôles, nombreux, dans la création d'une BD, même si le plus souvent le lecteur ne retient qu'un nom. En fait, la paternité des dialogues est ambivalente pendant les deux périodes identifiées par Glaude. Pendant une première période, il y avait beaucoup de collaborateurs à une seule BD ou même une seule planche; et ensuite pendant une deuxième période, on voit l'affirmation du rôle du scénariste qui avant, le plus souvent, n'était même pas mentionné au profit quasi exclusif du dessinateur. Après un rappel des conditions d'émergence du métier de scénariste dans la BD franco-belge, Glaude souligne l'influence jijéenne sur l'écriture des dialogues au journal de Spirou, «en partant du fait que Jijé réservait cette prérogative au dessinateur» (95). Franquin et Greg s'écartent de ce modèle jijéen développant ensemble un style novateur dans les dialogues de Spirou et Fantasio et se dégageant de l'héritage de l'école du studio de Jijé. Ce style novateur avait commencé avec « Modeste et Pompon » publié dans l'hebdomadaire concurrent, Tintin, entre 1955 et 1959, où la fonction des dialogues était de caractériser les personnages, non en tant qu'individus mais comme incarnant une classe sociale, alors que chez Jijé, ces dialogues 
caractérisaient l'action. « Après 'Modeste et Pompon', les Spirou et Fantasio de Franquin et Greg continuent d'accorder une priorité à la lisibilité » (106).

11 Après avoir rappelé les deux premières périodes de l'art franquinien (l'immédiat aprèsguerre « moelleux » [114] et la désinvolture des années 50), dans un langage parfois peu académique ("emmerde, pisse, ...»), mais approprié ici puisque Erwin Dejasse le reprend aux auteurs mêmes, rappelant le «joyeux mépris pour les règles du prétendu bon goût " (114) de Franquin, le critique fait une étude détaillée de la dernière BD de Spirou par Franquin, Panade à Champignac. Dans cet album célèbre, « Franquin se livre à une entreprise de déconstruction iconoclaste qui a souvent été rapprochée des Bijoux de la Castafiore » (116). Franquin a souvent ridiculisé ses " héros », comme le marsupilami qui a toujours été un protagoniste distant. Cela ressort d'autant plus dans cet album, où le personnage de "l'anarchiste » au visage caché, évoluant dans les marges, annonce qu'il n'y a pas de fin possible à cette histoire en dépit du "à suivre ", inscrit en fin d'album." (119). Après avoir plus ou moins participé à l'euphorie moderniste et consumériste des années 50-60, comme beaucoup dans la société qui l'entoure, Franquin montre "une sensibilité écologiste qui se précisera dans les créations ultérieures » (119). Et ce, même si l'artiste, comme beaucoup d'autres auteurs de sa génération venus à la BD quand elle était tout au plus un " passe-temps sympathique » (120), insiste qu'il n'est pas un dessinateur politique (120). Si Spirou est contemporain de l'underground (américain, français, avec Hara-Kiri, 1960, ou italien, avec Linus, 1965), il « reste largement imperméable au bouillonnement social et contre-culturel »(122), voire participe à la culture officielle. Franquin, lui, « vilipende un 'star system' dont il est, à son corps défendant, l'incontournable vedette » (126). On retrouvera cette idée dans le chapitre 11 de Hagelstein, qui met en évidence le fait qu'une lecture politique ne doit nullement s'embarrasser de l'avis de l'auteur. En ce sens, si Panade a souvent été comparé aux Bijoux d'Hergé, les deux auteurs ont abouti à deux positions diamétralement opposées: le conservatisme d'Hergé, qui aboutira à la mort de son personnage (dans L'Alph'art et de par l'interdiction de continuer ou renouveler ses aventures), s'oppose à la vivacité continue de Spirou comme les chapitres suivants le montrent à travers les reprises par Chaland (11), Bravo (12), et d'autres plus récentes.

Le chapitre 9 de Mélanie Tasset retrouve certaines des idées du chapitre précédent. Les chapitres suivant l'ordre chronologique, celui-ci continue de traiter de la «déshéroïsation » chez Franquin, avec dans ce cas, le personnage de Gaston Lagaffe. Tasset montre comment en 10 ans, de 1957 à 1967, date de parution de l'album Panade et de l'album 1 (en fait 5) Gare aux gaffes, Lagaffe, l'anti-Spirou, a progressivement remplacé Spirou dans la production et le cœur de Franquin. L'auteure conclut avec les « adieux de Franquin à son Spirou » (125) où l'on voit Spirou qui apparait souriant à côté de Fantasio hilare, tous deux étant en vacances sur la plage, et Lagaffe arrivant sur la route qui longe la plage et qui occupe à lui seul toute la dernière case horizontale.

Dans le chapitre 10, « D'un Z à l'autre » (137-148), Clément Lemoine s'attache à décrire l'itinéraire d'un personnage secondaire mais crucial du monde de Spirou. Car, contrairement à Spirou même, à Fantasio, à Champignac et au maire qui « sont toujours ramenés à leur identité propre » (137), Zorglub change dans ses 3 albums originels et dans les albums des repreneurs (Fournier ou Tome et Janry), ainsi que dans les « oneshots ». Avec sa première apparition dans Z comme Zorglub (1960), souvent considéré comme un des chefs d'œuvre de la série, il est déjà ambivalent et bien que rarement analysé politiquement (contrairement au cas du Dictateur et le champignon), il reflète le 
contexte historique du passé encore récent (e.g., Stalingrad/Zorgrad). Comparée à la technologie du savant fou de Champignac, celle de Zorglub incarne une technologie toute puissante et une modernité consommatrice qui cherche constamment à éliminer la mémoire humaine. Mais Zorglub reste fondamentalement ambivalent y compris dans les nombreuses reprises (dans au moins 6 albums et un film d'A. Coffe [sic, 148--Coffre], 2018) soit inscrites dans la série officielle, soit hors-série, et ce, jusqu'au plus récent ( $L a$ fille $d u \mathrm{Z}, 2017$ ) où la « paternité permet de laisser au savant ses qualités humaines sans nier sa dangerosité » (148).

Dans le chapitre 11, «Chaland nous tend un piège. Tentatives Spirou » (149-158), Maud Hagelstein montre que Chaland semble attirer les lecteurs par son style facile et ses références au monde ancien de l'âge d'or. Mais il les piège car il «le[s] met face aux personnages les plus vils" prônant des valeurs désuètes (catholicisme déviant, colonialisme persistant, racisme décomplexé [150]), « et sans doute le piège fonctionnet-il d'autant mieux que Chaland y a lui-même succombé » (150). Hagelstein nous donne une très belle lecture politique d'une œuvre elle-même politique mais souvent mal comprise. Cette incompréhension est sans doute due au fait que les milieux de la BD n'étaient pas prêts à accepter cette vision critique d'albums ancrés dans leur société non seulement par leur relation à la modernité (esthétique, technologique, consommatrice), mais aussi par leur relation problématique au colonialisme, au racisme ou au sexisme.

Dans le long et riche chapitre 12, intitulé «L'Archive Spirou revisitée » (159-176), Benoît Crucifix et Pedro Moura nous proposent une stimulante réflexion sur l'usage de l'archive par deux « repreneurs » de Spirou qui ont utilisé deux stratégies différentes. "[L]à ou Le Journal d'un ingénu procède d'une façon centripète, ordonnant les éléments de l'archive d'une façon clairement coordonnée et téléologique, recréant une histoire cohérente et prenant une certaine position critique par rapport à la tradition francobelge de $\mathrm{BD}$, le groom [vert-de-gris] se présente comme une succession de gags et de situations toutes faites, privilégiant le genre de l'aventure typique de Spirou mais doublé d'un surplus citationnel qui tourne le récit en un véritable vertige centrifuge " (174-75). Le chapitre, écrit de manière très précise et très claire, conjugue une réflexion sur la problématique de l'archive en général à travers l'exemple de son usage dans le domaine particulier de la bande dessinée, et des analyses détaillées et subtiles du contenu (textuel et visuel) et de la forme (narrative et esthétique) des deux albums. Faute de place, je ne donnerai qu'un exemple parmi ceux présentés par les auteurs du chapitre. Dans le Spirou de Bravo, le héros embrasse une jeune fille et cette expérience fait partie de «l'apprentissage lent et parfois douloureux de Spirou» (170). Mais dans le Groom-vert-de-gris, cette même expérience "relève d'une simple opportunité » (172). Cette vulgarisation se trahit d'ailleurs visuellement lorsque l'étoile que cette jeune fille juive porte quand elle est représentée dans une bulle-pensée se transforme dans la case suivante en étoile symbolisant l'étourdissement dû au choc physique.

Dans les chapitres précédents, certaines brèves allusions ont été faites à l'influence de Spirou sur d'autres BD, d'autres séries et dans d'autres médias (e.g., cinéma, ...). Dans le dernier chapitre (13), intitulé « $N$ comme cornichon: pas même un pastiche » (177-184), Laurent Dumoulin donne un exemple intéressant de l'influence que Spirou a eue à travers une œuvre réalisées par deux artistes marginaux ( aux lecteurs peu nombreux", 180) mais influents dans le monde de la BD, i.e., Rosse et Schlingo. Dumoulin nous montre comment ces deux « cornichons » d'auteurs parodient le texte. 
Même si Dumoulin au début rappelle aussi l'évidente allusion au cubisme, il aurait peut-être été utile d'ajouter que l'intérêt de cet album est alors aussi de faire se rencontrer ce que longtemps on a considéré le bas (la $\mathrm{BD}$, et ici une $\mathrm{BD}$ sérielle longtemps réservée aux enfants et adolescents, un média populaire; le bas du bas du bas) et le haut (l'Art contesté par des "Auteurs"; le haut du haut du haut au 20e siècle). Enfin, cet album publié aux Humanoïdes associés n'aura de sens que pour les amateurs de Spirou, ceux qui connaissent l'hypotexte, mais il révèle aussi qu'en 2018, la BD est bien devenue un art.

La brève conclusion (185-87) met en évidence que si une étude par auteur est encore intéressante il est aussi nécessaire de la compléter par des études de type plus macroscopique et/ou quantitatif: compléter des interprétations (point de vue herméneutique typique des Humanités) par des explications (perspective scientifique des sciences humaines et sociales). Comme souligné ci-dessus, les auteurs et les directeurs du volume ont régulièrement tissé des liens explicites ou implicites entre plusieurs albums et chapitres, ce qui d'une part montre qu'ils ont fait un bon travail de relecture, et compense l'absence de l'index (trop habituelle dans le monde de l'édition francophone). Cette conclusion se termine en reconnaissant qu'il reste encore beaucoup à faire, citant certains auteurs délaissés, en particulier des années 1970 (par exemple, Fournier, mais plus encore Broca et Cauvin à peine cités ici et là), le contexte éditorial (travail commencé par Pissavy-Yvernault), le projet pédagogique de l'éditeur catholique (en partie fait par Delisle, cité plusieurs fois dans ce volume), le côté transmédiatique, immense (du parc Spirou aux dessins animés, pas mentionnés ici), et son corollaire, le monde matériel de la BD avec les figurines, la publicité (à laquelle fait allusion Dejasse, 125), les jeux inclus dans les magazines, les jeux vidéo tirés des aventures, et l'étude des publics (à travers les lettres adressées au magazine mais aussi à travers des enquêtes de lecteurs). J'ajouterais qu'il manque cruellement et symptomatiquement une étude du genre (gender) dans le monde de Spirou (des personnages, des lectrices, des auteures). Si le genre est mentionné deux fois par Vrydaghs (50), rien n'est dit de Seccotine, ... Audrey, Kassandra, et d'autres femmes importantes ou secondaires.

18 Je voudrais encore mentionner un problème qui n'est sans doute pas dû aux auteurs mais à l'éditeur. S'il y a beaucoup d'illustrations, y compris en couleur, et souvent de bonne qualité, certaines images sont parfois de trop petite dimension, y compris lorsqu'il y assez d'espace blanc pour les présenter plus grandes comme par exemple la figure 5 de la page 110 et de la page 111. Ainsi, trouve-t-on une image presque illisible, et même « il-visible ", page 79, surtout celle de Toto qui est difficile d'accès.

19 Cependant mis à part ces quelques petits problèmes, l'ouvrage est remarquable et devrait se trouver dans toutes les bibliothèques universitaires, et inspirera d'autres chercheurs (amateurs et professionnels) pour continuer ces relectures. 